\title{
Parallel hydrogenation for the quantification of wetting efficiency and liquid solid mass transfer in a trickle-bed reactor
}

\author{
Arie Jan van Houwelingen \\ Willie Nicol ${ }^{\star}$
}

Department of Chemical Engineering, University of Pretoria, Pretoria, 0002, South Africa

${ }^{*}$ Corresponding author. Email address: willie.nicol@up.ac.za (Willie Nicol)

\begin{abstract}
A novel method for the measurement of wetting efficiency in a trickle-bed reactor under reaction conditions is introduced. The method exploits reaction rate differences of two first-order liquid-limited reactions occurring in parallel, to infer wetting efficiencies without any other knowledge of the reaction kinetics or external mass transfer characteristics. Using the hydrogenation of linearand isooctenes, wetting efficiency is measured in a $50 \mathrm{~mm}$ internal diameter, high pressure trickle bed reactor. Liquid-solid mass transfer coefficients are also estimated from the experimental conversion data. Measurements were performed for upflow operation, and two literature-defined boundaries of hydrodynamic multiplicity in trickle flow. Hydrodynamic multiplicity in trickle flow
\end{abstract}


gave rise to as much as $10 \%$ variation in wetting efficiency, and $10-20 \%$ variation in the specific liquid-solid mass transfer coefficient. Conversions for upflow operation were significantly higher than in trickle-flow operation, due to complete wetting and better liquid-solid mass transfer characteristics.

Topical Heading: reactors, kinetics, and catalysis

Keywords: trickle-bed reactors, liquid-solid mass transfer, wetting efficiency, multiphase reactors, hydrodynamics

\section{Introduction}

Packed bed reactors that process gas and liquid reactants are extensively utilised in industry, most notably in the petrochemical industry for hydroprocessing ${ }^{1,2}$. These reactors can be operated in gas-liquid cocurrent downflow (trickle flow), cocurrent upflow, or countercurrent flow. Due to flexibility in terms of throughput, gas-liquid downflow reactors are often preferred when large process streams are involved ${ }^{3,4}$. The hydrodynamics of trickle flow are rather complex, and upflow operation have been advocated for pilot-scale studies ${ }^{5}$. Existing studies on the comparison of upflow with trickle flow operation were summarised by Chaudari et al. $(2002)^{6}$, who advocated systematic studies comparing these two operating modes; especially since several studies show possible advantages of upflow operation above trickle flow operation.

For hydroprocessing purposes, hydrodynamic parameters that influence mass transfer rates in the liquid phase are of particular importance ${ }^{4,7}$. These rates are 
primarily affected by the external liquid-solid mass transfer coefficient and wetting efficiency. Evidence of the influence of these parameters in reaction study is long in existence ${ }^{2,8,9}$, and especially wetting efficiency received widespread attention in literature.

The most important measurement method is based on tracer measurements ${ }^{10,11,12}$, whereas several reaction methods were also suggested $^{13,14,15}$. These methods employ the additive model of Hartman \& Coughlin $(1972)^{13}$, which requires an accurate kinetic description of the reaction. Other methods require correlations for liquid-solid mass transfer to estimate wetting efficiency ${ }^{16,17}$. Recently, Baussaron et al. $(2007)^{18}$ generated an extensive amount of wetting efficiency data obtained from a colorimetric method, which was later expanded and correlated by Julcour-Lebigue et al. $(2009)^{19}$.

The overwhelming majority of liquid-solid mass transfer data in literature was obtained with either dissolution or an electrochemical technique. For the former, the packing material needs to be coated and is often not representative of a catalytic bed ${ }^{20}$, whereas for the latter the process fluid needs to be an electrolyte, limiting the applicability to typical process fluids ${ }^{21}$. There is a large deficiency of reactor-based measurements, especially at high pressures ${ }^{3}$.

In this work, a novel reaction method is presented for the measurement of wetting efficiency in a trickle-bed reactor. The method involves two reactions that are first order with respect to the non-volatile, limiting reagents, occurring in parallel throughout the reactor. It is shown how the conversions (and relative difference) of the two reactions can be used to measure wetting efficiency 
without any other knowledge of the reaction kinetics and liquid-solid mass transfer coefficients. Mass transfer coefficients are also estimated from conversion data. Unlike for the wetting efficiency measurements, these estimations rely on an assumption regarding the general relationship between mass transfer coefficients and liquid superficial velocity.

Several studies report hysteresis in trickle flow, which is commonly attributed to

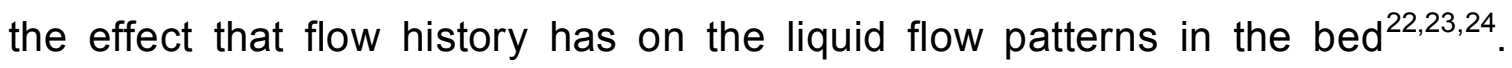
Although subject of numerous studies, trickle flow multiplicity studies focus almost exclusively on pressure drop, liquid holdup and flow texture ${ }^{23}$. Very few studies exist that attempt to quantify the effect of flow history or pre-wetting on wetting efficiency ${ }^{24}$ and liquid-solid mass transfer ${ }^{25}$. Moreover, direct studies of the effect of multiplicity on reactor performance are scarce ${ }^{26}$. In the current investigation, two of the pre-wetting methods as summarized by van der Merwe \& Nicol $(2009)^{27}$ are used to explore the boundaries of multiplicity behaviour.

Approximations of the reported parameters are based on packed bed conversion data for two reactions: Hydrogenation of linear octenes and hydrogenation of isooctenes (trimethylpentenes). This reaction system finds its application in the Fischer-Tropsch refining industry ${ }^{28}$. Fischer-Tropsch naphta contains up to $85 \%$ olefins, and requires severe hydrogenation. This leads to a drastic decrease in motor octane number (MON). The decrease in MON is highly dependent on the molecular structure of the hydrogenated olefin. As a rule, hydrogenation of linear olefins leads to a more severe drop in the octane number than the hydrogenation of branched olefins. It is therefore preferred to hydrogenate 
the branched olefins and retain the least branched olefinic molecules.

\section{Experimental}

\section{Trickle-bed reactor setup}

A flow sheet of the experimental setup is shown in figure 1. The setup is designed to provide for cocurrent gas-liquid upflow and downflow. The liquid reaction mixture, consisting of approximately $1 \%$ linear octene isomers and $2 \%$ isooctene isomers in a $\mathrm{C}_{14}-\mathrm{C}_{20}$ paraffin solvent is pumped with a Bran \& Luebbe $\mathrm{H} 2-31$ diaphragm metering pump, capable of delivering $70 \mathrm{~L} / \mathrm{min}$ at $80 \mathrm{bar}$. Estimated properties of the liquid feed are given in table 1. The liquid feed is preheated to the reaction temperature before entering a $50 \mathrm{~mm}$ i.d., $1000 \mathrm{~mm}$ length reactor. The reactor walls are temperature controlled using three external heaters with wall thermocouples. Eight internal thermocouples are used as illustrated in figure 2 to measure internal temperatures and verify isothermal operation. A Rosemount model 3051CD differential pressure transmitter is used for pressure drop measurements to check for flow stability. In downflow operation, gas and liquid is distributed through a distributor plate with twenty-one $4.5 \mathrm{~mm}$ holes, while liquid is distributed with $1 / 8$ " pipes that fits through these holes. In upflow operation, gas and liquid entering the bottom of the reactor is only distributed by a retaining sieve plate and the packing itself.

Nitrogen and hydrogen can be fed to the reactor, the flow rates being controlled by $0-30 \mathrm{NL} / \mathrm{min}$ Brookes mass flow controllers. Maximum operating pressure of the system is 80 bar. A water-cooled heat exchanger is installed in the product line to cool down the product to approximately $30^{\circ} \mathrm{C}$. Pressure is regulated with a back- 
pressure regulator and monitored at strategic points in the system with pressure indicators and transducers. Samples are taken in a sampling bomb with dip tube for gas-liquid separation. Based on the high boiling points of the liquid components, it is clear that evaporation and entrainment of liquid product in the gas will not significantly affect the product composition at $30^{\circ} \mathrm{C}$. The product stream can either be recycled to the feed tank or routed to the product tank.

\section{Experimental conditions and procedure}

For each experimental run, the olefins in the liquid feed was hydrogenated over a randomly packed bed of $0.3 \% \mathrm{Pd} / \mathrm{Y}-\mathrm{Al}_{2} \mathrm{O}_{3}$ spherical eggshell catalyst diluted with $\mathrm{y}-\mathrm{Al}_{2} \mathrm{O}_{3}$ supports. All particles have a diameter of $3 \mathrm{~mm}$ and a catalyst shell thickness of $0.3 \mathrm{~mm}$. All experiments were performed for five different liquid flow rates, corresponding to superficial velocities of $1.8,2.6,3.6,4.5$ and $7.5 \mathrm{~mm} / \mathrm{s}$; and three different flow configurations namely upflow, Levec pre-wetted trickle flow and extensively pre-wetted trickle flow. The start-up procedure for each type of flow configuration is as follows

- Upflow. The liquid flow is set to the required rate by adjusting pump stroke length and pump motor speed, and is fed to the bottom of the reactor, exiting at the top. Temperature control setpoints for the liquid feed pre-heaters and reactor heaters are set to the required temperatures. Nitrogen gas flow is introduced and the liquid is recycled to the feed tank until flow and temperature steady state has been reached. Once steady state is achieved, the product stream is rerouted to the product tank, nitrogen 
flow is shut off and hydrogen is introduced to the reactor. The feed tank is stirred, to ensure that the composition of the feed entering the reactor stays constant.

- Levec pre-wetted trickle flow. After the bed is flooded by feeding liquid in upflow, the liquid in the reactor is purged with nitrogen at atmospheric pressure, until no liquid can be detected in the reactor exit stream. The reactor is then pressurised with nitrogen to the required pressure, after which liquid is introduced to the top of the reactor at the required flow rate. It is ensured that the reactor pressure stays constant during the introduction of the liquid. The rest of the start-up procedure is the same as for upflow. For most hydrodynamic parameters, Levec pre-wetting represent the lower boundary of multiplicity in pre-wetted beds ${ }^{26}$.

- Extensively pre-wetted trickle flow. The reactor is flooded by feeding liquid at the required rate to the bottom of the reactor under recycle conditions, until no gas can be detected in the reactor exit stream. The liquid feed configuration is then changed from upflow to downflow, and nitrogen is introduced to the reactor. The rest of the start-up procedure is the same as for upflow. This pre-wetting procedure will in most cases result in operating on the upper boundary of the multiplicity envelope ${ }^{33}$.

Above start-up procedures require a measure for steady state, before nitrogen can be replaced by hydrogen. Steady state was verified by thermocouple readings, pressure drop, and flow rate measurements. Once temperature and pressure drop steady state is reached, the liquid product flow rate is repeatedly measured with a 
graduated cylinder and a stopwatch. If this stays constant, it is assumed that liquid holdup in the reactor stays constant and therefore that hydrodynamic stability has been reached. This takes between 10 and 20 system residence times, depending on the flow rate and configuration. For each experiment, at least $50 \%$ stoichiometric excess of hydrogen is fed to the reactor. With the highest conversions reported in this paper, this translates to 4.5 times the amount that has reacted. All downflow experiments were performed in the low interaction (trickle) flow regime. All experiments were performed at $60^{\circ} \mathrm{C}$ and 50 bar.

Two product samples were taken for each specific flow rate and configuration: The first sample is taken 10 reactor residence times (based on void volume) after achieving steady state, and the second 3-5 residence times later. The second sample serves to verify steady state conditions in the reactor.

Samples were analysed with an Agilent Technologies 6890 gas chromatograph (GC) fitted with a flame ionisation detector (FID). Elutriation was established on a $50 \mathrm{~m}$ long Pona column with a $0.2 \mathrm{~mm}$ inner diameter and a $0.5 \mathrm{~mm}$ film thickness with $\mathrm{N} 2$ as carrier gas at a flow rate of $25 \mathrm{ml} / \mathrm{min}$. A split ratio of 100:1 was used. The initial column temperature was $40^{\circ} \mathrm{C}$, where it was held for 5 minutes. Then the temperature was ramped for 15 minutes at $4^{\circ} \mathrm{C} / \mathrm{min}$ to obtain good separation of the $C_{8}$ reagents, after which the temperature is increased to $300^{\circ} \mathrm{C}$ at $25^{\circ} \mathrm{C} / \mathrm{min}$.

The catalyst bed consisted of a $630 \mathrm{~mm}$ of $70 \mathrm{~g}$ catalyst diluted with inert $\mathrm{Al}_{2} \mathrm{O}_{3}$ supports, packed between two layers of $140 \mathrm{~mm}$ of inert supports at the entrance and exit of the reactor. For a conversion of $X \leq 0.6$, the dispersion criterion of Sie 
\& Krishna (1998) suggest a minimum reactor length of $550 \mathrm{~mm}$ for dispersion to be negligible in all modes of operation if the reaction is first order. Both the catalyst and support were supplied by Hereaus. Particle density was $\pm 1100 \mathrm{~kg} / \mathrm{m}^{3}$. Bed porosity was $\varepsilon \approx 0.4$ for all experiments.

\section{Gas mass transfer resistances and reaction order}

Though the bulk of the reaction experiments were performed at the conditions stated above, two other sets of experiments were also conducted. First of all, it had to be ensured that the liquid entering the bed is saturated with hydrogen, independent of liquid flow rate and flow configuration. For all experiments, 140 $\mathrm{mm}$ of inert supports were used to provide for hydrogen saturation before entering the bed. That this amount of support is indeed enough to ensure saturation was verified experimentally: Two experimental runs were performed, one with an undiluted $(70 \mathrm{~g})$ catalyst bed situated $140 \mathrm{~mm}$ from the top reactor inlet and another with the bed situated close to the bottom of the reactor (the depth of the bed was $715 \mathrm{~mm}-775 \mathrm{~m}$ ). The available area for gas-liquid mass transfer before entering the catalyst bed is far more in the former than in the latter case for gasliquid upflow, and vice versa for trickle flow. Results for linear octane hydrogenation are shown in figure 3 . Since these two runs agree satisfactorily for all experimental conditions, it can be assumed that the liquid is saturated with the gas before entering the bed. Both experiments were repeated with good repeatability.

In another set of experiments, conversion data for the hydrogenation of a $1 \%$ 
linear octenes and $2 \%$ isooctenes feed was compared with hydrogenation of $0.5 \%$ linear octenes and $0.5 \%$ isooctenes. Results are shown in figure 4. Close agreement between the results suggests both reactions are liquid-limited and first order with respect to the liquid reagents: Should gas mass transfer resistances play a role, conversions for the lower concentration feed would be higher than for the more concentrated feed. Hence, it can be assumed that the partial pressure of hydrogen was constant throughout the bed for all experiments, so that pseudo-first order kinetics with respect to the liquid reagents can be assumed. Also, conversions that are independent of inlet concentration are characteristic for first order reactions.

\section{Results and Discussion}

\section{Conversion data}

Typical conversion data for an experimental run is shown in figure 5 . In the rest of the discussion an "experimental run" will refer to two conversion data points for both reactions at five different liquid flow rates for all three different modes of operation. All the datapoints from an experimental run were generated consecutively (in no specific order) without interruption of the reactor temperature. Conversion data for the two different reactions are of course generated in parallel. The lower conversion data in figure 5 is for isooctene hydrogenation, which is considerably slower than the hydrogenation of linear octenes. In total, nine experimental runs were performed, each consisting of a total of 60 conversion measurements (30 product samples for 15 different flow conditions and two 
reactions).

At all liquid flow rates and for both reactions, conversion decreases in the order upflow - extensively pre-wetted trickle flow - Levec pre-wetted trickle flow at the same liquid flow rate. Although both reactions were established to be liquidlimited and first order in terms of the olefin concentration, none of the data show good first order behaviour for the fastest reaction and conversion rates increase with liquid flow rate. For the slower reaction (isooctene hydrogenation), the upflow conversion data approximates first order behaviour. These observations are clear indicators of liquid-solid mass transfer resistances, since the effective reaction rate increases with liquid flow rate, and deviations from first order behaviour are more severe for the fast reaction. Hence, the following reactor model will be used in the treatment of upflow conversion data:

$$
\begin{gathered}
-\ln (1-X)=\frac{k_{T} V_{c a t}}{Q_{L}} \\
k_{T}=\frac{k_{R} k_{L S} a}{k_{R}+k_{L S} a} \\
\text { Where } a=\frac{6}{d_{p}} ; V_{c a t}=\frac{m_{c a t}}{\rho_{c a t}} ; k_{R}=\eta_{p} k_{r}
\end{gathered}
$$

The rate constant $k_{R}$ in equation (2) includes the particle efficiency factor for a fully wetted particle, as is shown in equation (3). Where upflow conversion data for the slower reaction approximates first order behaviour, significant deviations still persist in trickle flow at low liquid flow rates. The deviations from first order behaviour, even for the slower reaction, will be interpreted as a combined effect of resistance to mass transfer and incomplete wetting. If it is assumed that the area for 
liquid-solid mass transfer and the particle reaction rate (internal diffusion incorporated) is linearly dependent on the wetting efficiency, the apparent first order rate constant is given by:

$$
\begin{aligned}
k_{T} & =\frac{\left(f \cdot k_{R}\right)\left(k_{L S} a \cdot f\right)}{f \cdot k_{R}+k_{L S} a \cdot f} \\
& =f \frac{k_{R} k_{L S} a}{k_{R}+k_{L S} a}
\end{aligned}
$$

The assumption of linearity between the particle efficiency factor and wetting efficiency requires generalized particle moduli larger than $3^{7}$. For all experiments, the modulus for the fast reaction was determined to be larger than 10 based on shell volume, and larger than 2.6 for the slower reaction. For this modulus, the maximum error in assuming a linear dependence of the particle efficiency on wetting efficiency is less than $1 \%$.

Equation (4) will be used in the treatment of trickle flow conversion data. Note that for the rest of the discussion, $\mathrm{k}_{\mathrm{T}}$ will be specific to each conversion datapoint. For example, $k_{L S}$ and $f$ are dependent on the hydrodynamics (i.e. upflow/downflow, liquid flow rate and employed pre-wetting procedure). The liquid-solid mass transfer coefficient, $\mathrm{k}_{\mathrm{LS}}$, as used in equation (4), is therefore not the same as in equation (3). The only parameters in equation (4) that are independent of hydrodynamic conditions are $k_{R}$, which is specific to each reaction, and $a$, that is a function of the packing properties only.

\section{Data refinement}

Although all characteristics of figure 5 were highly repeatable for most of the 
experimental runs, only a few experimental runs were quantitavely repeatable. An example of how conversion data varied from experimental run to experimental run is shown in figure 6 . The large scatter is attributed to differences in catalyst activity. Two types of activity variations are possible: One where the catalyst activity varied within a run, and another where the catalyst was stable during a run, but at a different activity than during other experimental runs. Data from the former type of activity variation can not be used, whereas data from the latter type can still be useful if treated correctly.

For selection of useful conversion data, it is first of all necessary to discard all data from experimental runs during which the catalyst deactivated: Catalyst deactivation while performing an experimental run might influence the interpretation of hydrodynamics. For indication of catalyst stability during an experimental run, the following catalyst activity indicator (CAI) was defined, which can be calculated from conversion data without any knowledge of the reaction rate constants (using the expression on the right):

$$
C A I=\frac{k_{R 1} \cdot k_{R 2}}{k_{R 1}-k_{R 2}}=\left.\frac{k_{T 1} \cdot k_{T 2}}{k_{T 1}-k_{T 2}}\right|_{\text {upflow }}
$$

The derivation of above equation is shown in equations 7 and 8 , where it is used for the estimation of wetting efficiency. For complete wetting in the upflow mode, the CAI should be independent of liquid flow rate under liquid-limited conditions, and is directly related to the catalyst activity. All experimental runs during which the CAI decreased notably were discarded. An example of how the CAl is used is shown in figure 7. 
Because of catalyst deactivation, data from four out of the nine experimental runs had to be discarded. Although all of the retained datasets are generated with stable catalyst, the stable catalyst activity varied from experimental run to experimental run as is seen in figure 8. It is therefore important to develop methods for the estimation of the hydrodynamic parameters in equations (1) and (4) that are insensitive to the specific catalyst activity.

\section{Wetting efficiency}

Consider two first order reactions with particle rate constants $k_{R 1}$ and $k_{R 2}$ occurring in a trickle bed reactor as modeled in equation (4). Using the effective rate constants $\mathrm{k}_{\mathrm{T} 1}$ and $\mathrm{k}_{\mathrm{T} 2}$ obtained from conversion data, the liquid mass transfer coefficient can be calculated twice for known reaction rate constants and wetting efficiency:

$$
k_{L S} a=\frac{k_{T 1} k_{R 1}}{f \cdot k_{R 1}-k_{T 1}}=\frac{k_{T 2} k_{R 2}}{f \cdot k_{R 2}-k_{T 2}}
$$

Note that equation (6) is only valid if both reactions take place under the same hydrodynamic conditions, and refer to the treatment of one specific conversion datapoint. The relationship also relies on the assumption that the molecular

diffusivities of both reagents are the same. According to the Wilke-Chang correlation, this assumption holds true for the current system (see table 1).

By rearranging equation (6), it is possible to calculate wetting efficiency at a specific hydrodynamic state (mode of operation and liquid flow rate) if $k_{R 1}$ and $\mathrm{k}_{\mathrm{R} 2}$ is known. 


$$
f=\underbrace{\frac{k_{R 1}-k_{R 2}}{k_{R 1} k_{R 2}}}_{A} \times \underbrace{\frac{k_{T 1} k_{T 2}}{k_{T 1}-k_{T 2}}}_{B}
$$

Part (A) of equation (7) contains only reaction rate constants and is constant for a stable catalyst. Therefore, part (B) of equation (7) is directly proportional to wetting efficiency and should be constant during upflow operation if the assumption of complete wetting in upflow holds true:

$$
\frac{k_{R 1}-k_{R 2}}{k_{R 1} k_{R 2}}=\left.\frac{k_{T 1}-k_{T 2}}{k_{T 1} k_{T 2}}\right|_{\text {upflow }}
$$

Compare equation (8) to the definition of the CAI in equation (5). It was found that the CAl is a constant for stable catalyst or a function of time-onstream only for an unstable catalyst as is shown in figure 7 . Therefore, the wetting efficiency in upflow operation is constant and independent of liquid flow rate, and the assumption of complete wetting holds true. Wetting efficiencies in trickle flow operation can therefore be calculated if conversion data is available for upflow operation at the same catalyst activity.

$$
f_{T B R}=\left.\frac{k_{T 1} k_{T 2}}{k_{T 1}-k_{T 2}}\right|_{T B R} \times\left.\frac{k_{T 1}-k_{T 2}}{k_{T 1} k_{T 2}}\right|_{\text {upflow }}
$$

Note that for the calculation of wetting efficiency, no knowledge of the kinetic rate constants $k_{R 1}$ and $k_{R 2}$ is required, and it is possible to calculate wetting efficiency from the raw conversion data as long as upflow conversion data for only and any one liquid flow rate is available at the same catalyst activity, i.e. the catalyst was stable during an experimental run. It is not necessary to have upflow conversion data available at all liquid flow rates: only one upflow conversion 
datapoint for both reactions is needed to calculate the quantity defined in equation (8), as long as the catalyst is stable. Figure 9 shows wetting efficiencies in trickle flow operation as calculated with equation (9). The averaged values for all experimental runs with stable catalyst are shown in figure 10. As expected, hydrodynamic multiplicity is the most severe at low liquid velocities $( \pm 10-15 \%$ variation), where liquid flow in Levec pre-wetted beds tend to channel ${ }^{24}$. Wetting efficiency results for the Levec pre-wetted operation agree well with the correlation of Julcour-Lebigue et al. (2009) ${ }^{19}$. The experimental data that was used in this correlation was generated in Levec pre-wetted beds.

\section{Liquid-solid mass transfer}

Contrary to the estimation of wetting efficiency, approximations of kinetic rate constants $k_{R 1}$ and $k_{R 2}$ are needed to estimate mass transfer rates from conversion data. For constant temperature, fluid properties, reagent diffusivity and bed properties, most mass transfer correlations have the following functional relationship with liquid flow rate ${ }^{3}$ :

$$
\mathrm{k}_{L S} a=k_{0} Q^{k 1}
$$

Based on this relationship the apparent rate constant at a specific liquid flow rate in upflow operation will, according to equation (4), be equal to: 


$$
\begin{aligned}
& \mathrm{k}_{T x, i j}=\frac{k_{R x, i} k_{0} Q_{j}^{k_{1}}}{k_{R x, i}+k_{0} Q_{j}^{k_{1}}} \\
& \begin{aligned}
& \text { Where } x=1 \text { for linear octene hydrogenation } \\
& x=2 \text { for isooctene hydrogenation } \\
& i \quad \text { refers to a specific experimental run } \\
& j \quad \text { refers to the liquid flow rate }
\end{aligned}
\end{aligned}
$$

The coefficients $k_{0}$ and $k_{1}$ should be independent of the reaction rates, and the following function was minimised in order to obtain approximations of (a) kinetic rate constants for both reactions $\mathrm{x}$ and all experimental datasets $\mathrm{i}$, and (b) liquidsolid mass transfer for upflow operation as a function of liquid flow rate:

$$
F=\sum_{x i j}\left|X_{x i j}+\exp \left(\frac{k_{R x, i} k_{0} Q_{j}^{k_{1}-1} \cdot V_{c a t}}{k_{R x, i}+k_{0} Q_{j}^{k_{1}}}\right)-1\right|
$$

Minimisation of this function is an iterative procedure, where $k_{R, x i}$ is fitted onto conversion dataset $i$ specific to reaction $x$ with set values for $k_{0}$ and $k_{1}$ (1 parameter fitted to \pm 10 datapoints), and $k_{0}$ and $k_{1}$ is fitted to all conversion datasets with $k_{R, x i}$ set for each dataset/reaction (2 parameters fitted to \pm 100 datapoints). Figure 11 shows datafits obtained with this procedure. Estimated values for $k_{R 1}$ and $k_{R 2}$ vary between 0.11 and 0.05 , and 0.015 and $0.01 \mathrm{~s}^{-1}$ respectively, based on catalyst volume.

Now that the particle kinetic rate constants are known, mass transfer coefficients can be calculated for all flow rates and operating modes by substituting equation (7) into equation (6):

$$
\mathrm{k}_{L S} a=\frac{k_{T 1}-k_{T 2}}{k_{T 2} / k_{R 2}-k_{T 1} / k_{R 1}}
$$

With the wetting efficiency results from the previous section, it is also possible 
to calculate mass transfer coefficients directly with equation (6). Equation (13) is preferred, so that mass transfer rates can be calculated without making use of the wetting efficiency results. Liquid-solid mass transfer coefficients calculated with equation (13) are independent of the wetting efficiency and an indication of the specific rate of mass transfer at any specific point in the bed. Most liquid-solid mass transfer studies in trickle-beds are based on either a dissolution method ${ }^{20,34,35,36}$ or an electrochemical method ${ }^{25,37,38,39,40}$. These experimental methods lead to mass transfer coefficient measurements that include wetting efficiencies, i.e., usually $k_{\llcorner S}$ $\times f$ is measured. To calculate $k_{L S} \times f$, one can once again use equations (6) and (7) to find the following relationship:

$$
\mathrm{k}_{L S} a . f=\frac{k_{R 1}-k_{R 2}}{k_{R 1} / k_{T 1}-k_{R 2} / k_{T 2}}
$$

For upflow where $f=1$, equation (13) and (14) should yield the same results, which can be used as a test whether the estimated reaction rate constants are reasonable. That this is indeed the case is shown in figure 12 , which is a parity plot of upflow mass transfer rates calculated via equation (13) and via equation (14). Wetting efficiency-based ( $k_{\llcorner S} f$, equation 14$)$ and specific ( $k_{L S}$, equation 13$)$ liquidsolid mass transfer coefficients for trickle-bed operation are shown in figure 13. Reported values are averages of 5 measurements. Overall, hydrodynamic multiplicity gave rise to about $10-20 \%$ variation in $\mathrm{kLS}_{\mathrm{LS}}$. Literature correlations for dissolution-based ${ }^{34}$ and electrochemical-based ${ }^{39}$ mass transfer rate measurements are also shown on the figure. The latter is recommended by Dudukovic et al. $(2002)^{3}$ for trickle-bed design purposes. However, many correlations predict liquidsolid mass transfer coefficients as much as ten times smaller than reported in the 
figure.

Multiplicity of liquid-solid mass transfer in trickle beds has previously been explained as a combined liquid holdup-wetting efficiency effect ${ }^{26}$ : At a specific superficial liquid velocity, a low liquid holdup should enhance mass transfer due to higher interstitial liquid velocities. On the other hand, low wetting efficiencies should be detrimental for mass transfer. That liquid holdup (interstitial velocity) and wetting efficiency (area for mass transfer) are not the only hydrodynamic properties that influence mass transfer rates, is clear from the inset in figure 13. Though the instantaneous mass transfer coefficients in this subfigure are not affected by wetting efficiency, a marked difference between Levec and extensively pre-wetted beds still persist. This finding is in direct agreement with the results from Joubert \& Nicol (2009) ${ }^{41}$ who observed slower liquid-solid mass transfer in a Levec pre-wetted bed than in an extensively pre-wetted bed, even though the interstitial velocity is higher (lower liquid holdup). This suggest that the difference in flow structure between the Levec and extensively pre-wetted beds ${ }^{24,42,43,44,45}$ has a severe impact on the liquid-solid mass transfer characteristics.

Lastly, liquid-solid mass transfer in trickle flow operation is compared to mass transfer in upflow operation in figure 14. Mass transfer coefficients in upflow are 12 to $30 \%$ higher for upflow operation than for trickle-flow operation at the same superficial liquid velocity, confirming that some flow characteristics in the trickle flow regime are detrimental for overall liquid-solid mass transfer rates. From the inset in Figure 14 it can be seen that the same trend applies for the specific mass transfer coefficient. The difference would have been more severe if 
interstitial velocity was used instead of superficial velocity, but due to the lack of holdup data, quantification could not be performed.

\section{Conclusions}

A novel parallel first order reaction method was introduced to infer wetting efficiency in a trickle-bed reactor from conversion data of two liquid-limited reactions taking place in parallel in the reactor. The method is illustrated and validated by means of the parallel hydrogenation of linear and isooctenes in a high pressure, $50 \mathrm{~mm}$ i.d. trickle bed reactor. Where previous reactor-based wetting efficiency measurement methods require an accurate estimation of the reaction rate constant(s), the current method only requires the reactions to be liquid-limited and first order. The exact magnitudes of the rate constants are of lesser importance, so that wetting efficiency measurements are insensitive to variations in catalyst activity. The same equations that are used to calculate wetting efficiencies can even be used to monitor catalyst stability. Wetting efficiency results were realistic and in agreement with literature. Liquid-solid mass transfer coefficients were also determined from the conversion data, by assuming a functional relationship between the liquid flow rate and liquid-solid mass transfer that is often encountered in literature. Two different pre-wetting procedures for trickle flow were investigated, in order to explore the boundaries of hydrodynamic multiplicity. Trickle flow results were also compared to upflow operation. The trickle flow multiplicity envelope shows up to $10 \%$ variation in wetting efficiency and $10-20 \%$ variation in mass transfer rates. Results suggest that different flow 
morphologies in trickle flow, that can have different effects on liquid-solid mass transfer. Overall, conversions for upflow were substantially higher than for trickle flow operation, due to complete wetting and better specific liquid-solid mass transfer characteristics.

\section{Acknowledgements}

Sasol Research \& Development and the National Research Foundation of South Africa are gratefully acknowledged for their financial support.

\section{Nomenclature}

a

CAI

$d_{p}$

$f$

$\eta_{\mathrm{p}}$

$\mathrm{k}_{0}$

$\mathrm{k}_{1}$

$\mathrm{k}_{\mathrm{r}}$

$k_{R}$
Particle specific surface area $\left(a=6 / d_{p}\right), 1 / m$

Catalyst activity indicator, defined in equation (5)

Particle diameter, $\mathrm{m}$

Wetting efficiency

Pellet efficiency factor (for a fully wetted particle)

Fitting constant for upflow mass transfer (eq. 10)

Fitting constant for upflow mass transfer (eq. 10)

Intrinsic first order kinetic rate constant, based on

particle density $1 / \mathrm{s}$

First order particle kinetic rate constant based on 


\begin{tabular}{|c|c|}
\hline $\mathrm{k}_{\mathrm{T}}$ & Apparent first order rate constant $1 / \mathrm{s}$ \\
\hline $\mathrm{k}_{\mathrm{LS}}$ & Liquid-solid mass transfer coefficient $\mathrm{m} / \mathrm{s}$ \\
\hline $\mathrm{m}_{\mathrm{cat}}$ & Catalyst mass, $\mathrm{g}$ \\
\hline MON & Motor octane number \\
\hline$Q_{L}$ & $\begin{array}{l}\text { Liquid flow rate, } \mathrm{ml} / \mathrm{min} \text { (in figures) or } \mathrm{ml} / \mathrm{s} \text { (in } \\
\text { equations) }\end{array}$ \\
\hline$\rho_{\text {cat }}$ & Catalyst particle density, $\mathrm{g} / \mathrm{ml}$ \\
\hline$V_{\text {cat }}$ & Total catalyst volume, $\mathrm{ml}$ \\
\hline$v_{S G}$ & Gas superficial velocity, $\mathrm{cm} / \mathrm{s}$ \\
\hline $\mathrm{V}_{\mathrm{SL}}$ & Liquid superficial velocity, $\mathrm{mm} / \mathrm{s}$ \\
\hline$x$ & Conversion \\
\hline z & Bed depth, mm \\
\hline \multicolumn{2}{|c|}{ Subscripts } \\
\hline 1 & Refers to linear octene hydrogenation \\
\hline 2 & Refers to isooctene hydrogenation \\
\hline $\mathrm{i}$ & Refers to experimental run with stable catalyst \\
\hline j & Refers to specific liquid flow rate \\
\hline$x$ & Refers to specific reaction \\
\hline
\end{tabular}




\section{References}

1. Gianetto A, Specchia V. Trickle-bed reactors: State of art and perspectives. Chemical Engineering Science. 1992;47:3197-3213.

2. Satterfield CN. Trickle bed reactors. AIChE Journal. 1975;21:209-228.

3. Dudukovic MP, Larachi F, Mills PL. Multiphase catalystis reactors: A perspective on current knowledge and future trends. Catalysis Reviews. 2002;44:123-246.

4. Sie ST, Krishna R. Process development and scale up: III. Scale-up and scale-down of trickle bed processes. Reviews in Chemical Engineering. 1998;14:203-252.

5. De Wind M, Platenga FL, Heinerman JJL, Homanfree HW. Upflow versus downflow testing of hydrotreating catalysts. Applied Catalysis. 1988;43:239-252.

6. Chaudari RV, Jaganathan $R$, Mathew SP, Julcour $C$, Delmas $H$. Hydrogenation of 1,5,9-cyclodecatriene in fixed-bed reactors: Down- vs. upflow modes. AIChE Journal 2002;48:110-125.

7. Dudukovic MP. Catalyst effectiveness factor and contacting efficiency in trickle-bed reactors. AIChE Journal. 1977;23:940-944.

8. Henry HC, Gilbert JB. Scale up of pilot plant data for hydroprocessing. Industrial Engineering Chemistry Process Design and Development. 1973;12:328-334. 
9. Sedriks W, Kenney CN. Partial wetting in trickle bed reactors the reduction of crotonaldehyde over a palladium catalyst. Chemical Engineering Science. 1973;28:559-568.

10.Schwartz, TG, Wegwe, E, Dudukovic, MP. A new tracer method for determination of liquid-solid contacting effectiveness in trickle-bed reactors. AIChE Journal. 1976;22:894.

11. Colombo AJ, Baldi G, Sicardi S. Solid-liquid contacting effectiveness in trickle-bed reactors. Chemical Engineering Science. 1976;31:1101-1108.

12. Mills PL, Dudukovic MP. Evaluation of liquid-solid contacting by tracer methods. AIChE Journal. 1981;27:893-903.

13. Hartman M, Coughlin, RW. Oxidation of ethanol in gas-liquid cocurrent upflow and downflow reactors. Chemical Engineering Science. 1972;27:867-880.

14. Ruecker CM, Ackgerman. Determination of wetting efficiencies for a trickle bed reactor at high temperature and pressure. Industrial Engineering Chemistry Research. 1987;26:164-166.

15.Llano JJ, Rosal R, Sastre H, Diez FV. Determination of wetting efficiency in trickle-bed reactors by a reaction method. Industrial Engineering Chemistry Research. 1997;36:2616-2625.

16. Mata A, Smith JM. Transport processes in multiphase reactor systems. AIChE Symposium Series. 1981;77:29-35

17. Goto S, Mabuchi K. Oxidation of ethanol in gas-liquid cocurrent upflow and 
downflow reactors. 1984;62:865-869.

18. Baussaron L, Julcour-Lebigue C, Wilhelm A, Boyer C, Delmas H. Partial wetting in trickle-bed reactors: Measurement techniques and global wetting efficiency. 2007;46:8397-8405.

19. Julcour-Lebigue C, Augier F, Maffre H, Wilhelm A, Delmas H. Measurements and Modeling of Wetting Efficiency in Trickle-Bed Reactors: Liquid Viscosity and Bed Packing Effects. Industrial Engineering Chemistry Research. 2009;48:68116819.

20.Specchia V, Baldi G, Gianetto A. Solid-liquid mass transfer in concurrent twophase flow through packed beds. Industrial Engineering Chemistry Process Design and Development. 1978;17:362-367.

21. Latifi MA, Laurent A, Storck A. Liquid-solid mass transfer in a packed bed with downward cocurrent gas-liquid flow: An organic liquid phase with high Schmidt number. The Chemcial Engineering Journal. 1988;38:47-56.

22. Kuzeljevic ZV, van der Merwe W, Al-Dahhan MH, Dudukovic MP, Nicol W. Effect of operating pressure on the extent of hysteresis in a trickle bed reactor. Industrial Engineering Chemistry Research. 2008;47:7593-7599.

23. Maiti R, Khanna R, Nigam KDP. Hysteresis in trickle-bed reactors: A review. Industrial Engineering Chemistry Research. 2006;45:5185-5198.

24. Van Houwelingen AJ, Sandrock C, Nicol W, Particle wetting distribution in trickle bed reactors. AIChE Journal. 2006;52:3532-3542. 
25. Sims WB, Schulz FG, Luss D. Solid-liquid mass transfer to hollow pellets in a trickle bed, Industrial Engineering Chemistry Research. 1993;32:1895-1903.

26. Van der Merwe W, Nicol W, Al-Dahhan MH. Effect of hydrodynamic multiplicity on trickle bed reactor performance. AIChE Journal. 2008;54:249-257.

27. Van der Merwe W, Nicol W. Trickle flow hydrodynamic multiplicity: Experimental observations and pore-scale capillary mechanism. Chemical Engineering Science. 2009;64:1267-1284.

28. De Klerk A. Hydroprocessing peculiarities of Fischer-Tropsch syncrude. Catalysis Today. 2008;130: 439-445.

29. Van Velzen D, Cardozo RL, Langenkamp H. A liquid viscosity-temperaturechemical constitution relation for organic compounds. Industrial Engineering Chemistry Fundamentals. 1972;11:20-25.

30. Kendall J. The viscosity of liquids. II. The viscosity-composition curve for ideal liquid mixtures. Journal of the American Chemical Society. 1917:39;1787-1802.

31. Sugden S. A relation between surface tension, density, and chemical composition. Journal of the Chemical Society Transactions. 1924;25:1177-1189.

32. Wilke CR, Chang P. Correlation of diffusion coefficients in dilute solutions. AIChE Journal.1955:1:264.

33. Loudon DS, van der Merwe W, Nicol W. Multiple hydrodynamic states in trickle flow: Quantifying the extent of pressure drop liquid holdup and gas-liquid mass transfer variation. Chemical Engineering Science. 2006;61:7551-7562. 
34. Dharwarkar A, Sylvester ND. Liquid-solid mass transfer in packed beds. AIChE Journal. 1977;23:376-378.

35. Lakota A, Levec J. Solid-liquid mass transfer in packed beds with cocurrent downward two-phase flow. AIChE Journal. 1990;36:1444-1448.

36. Sylvester ND, Pitayagulsarn P. Mass transfer for two-phase cocurrent downflow in a packed bed. Industrial Engineering Chemistry Process Design and Development. 1975;14:421-426.

37. Chou TS, Worley FL, Luss D. Local particle-liquid mass transfer fluctuations in mixed phase cocurrent downflow through a fixed bed in the pulsing regime. Industrial Engineering Chemistry Research. 1979;18:279-283.

38. Hirose T, Mori Y, Sato Y. Liquid-to-particle mass transfer in fixed bed reactor with cocurrent gas-liquid downflow. Journal of Chemical Engineering of Japan. $1976 ; 9: 220-225$.

39. Latifi MA, Naderifar A, Midoux N. Experimental investigation of the liquid/solid mass transfer at the wall of a trickle-bed reactor-influence of Schmidt number. Chemical Engineering Science. 1997;52:4005-4011.

40. Trivizadakis ME, Karabelas AJ. A study of local liquid/solid mass transfer in packed beds under trickling and induced pulsing flow, Chemical Engineering Science. 2006;61:7684-7696.

41. Joubert R, Nicol W. Multiplicity Behavior of Trickle Flow Liquid-Solid Mass Transfer. Industrial Engineering Chemistry Research. 2009;48:8387-8392. 
42. Kan KM, Greenfield PF. Pressure drop and holdup in two-phase cocurrent trickle flows through beds of small packings. Industrial Engineering Chemistry Process Design and Development. 1979;18:740-745.

43. Lutran PG, $\mathrm{Ng} \mathrm{KM}$, Delikat, EP. Liquid distribution in trickle beds. An experimental study using computer-assisted tomography. Industrial Engineering Chemistry Research. 1991;30:1270-1280.

44. Ravindra PV, Rao DP, Rao MS. Liquid flow texture in trickle-bed reactors: An experimental study. Industrial Engineering Chemistry Research. 1996;36:51335145.

45. Van der Merwe W, Nicol W, de Beer F. Trickle flow distribution by X-ray tomography. Chemical Engineering Journal. 2007;132:47-59. 


\section{Figure Captions}

Figure 1. Schematic of the trickle-bed facility

Figure 2. Reactor detail

Figure 3. Test for saturation of liquid with hydrogen before entering the catalyst bed. The quantity $z$ refers to the position in the bed as measured from the top.

Figure 4. Conversion of linear octenes for feed concentrations of $0.5 \%$ and $1 \%$ linear octenes. The feed also contained $0.5 \%$ and $2 \%$ isooctenes, respectively.

Figure 5. Typical conversion versus flow rate dataset for an experimental run.

Figure 6. Unrefined upflow conversion data for the hydrogenation of linear octenes.

Figure 7 (a). An example of an experimental run for which the CAI indicates a drop in catalyst activity. All data generated during this run was discarded.

Figure 7 (b). An example of an experimental run with stable catalyst. The dataset generated during this run can be used. 
Figure 8. Upflow linear octene conversion data from experimental runs with stable catalyst.

Figure 9. Wetting efficiencies as calculated from conversion data with equation (9) as a function of liquid superficial velocity. Dotted lines indicate the estimations of wetting efficiency by Satterfield $(1975)^{2}$. (a) Extensively pre-wetted trickle flow. (b) Levec pre-wetted trickle flow.

Figure 10.Averaged wetting efficiency for trickle flow operation as a function of liquid superficial velocity.

Figure 11. Fits of upflow conversion data obtained from minimising equation (12). The highest and lowest activity cases are shown.

Figure 12. Parity plot for kısa.f and kısa for upflow operation. Good agreement confirms reasonability of estimated values for $k_{R 1}$ and $k_{R 2}$.

Figure 13. Averaged wetting efficiency-based liquid-solid mass transfer coefficients for trickle flow operation. Inset: Specific mass transfer coefficients.

Figure 14. Comparison of liquid-solid mass transfer in trickle-flow and upflow operation. Inset: Specific mass transfer coefficients. 


\section{Tables}

Table 1. Liquid feed properties

\begin{tabular}{|l|l|l|}
\hline Property & Estimated value & Estimation method \\
\hline Viscosity & $1.71 \mathrm{mPa} . \mathrm{s}$ & $\begin{array}{l}\text { Van Velzen et al. }(1972)^{29} \\
\text { Kendall }(1917)^{30}\end{array}$ \\
\hline $\begin{array}{l}\text { Surface tension } \\
\text { in solvent } \\
\text { Linear octenes } \\
\text { Iso-octenes }\end{array}$ & $27 \mathrm{mN} / \mathrm{m}$ & Sugden $(1924)^{31}$ \\
\hline Average molar mass & $1.13 \times 10^{-9} \mathrm{~m}^{2} / \mathrm{s}$ & \\
\hline
\end{tabular}




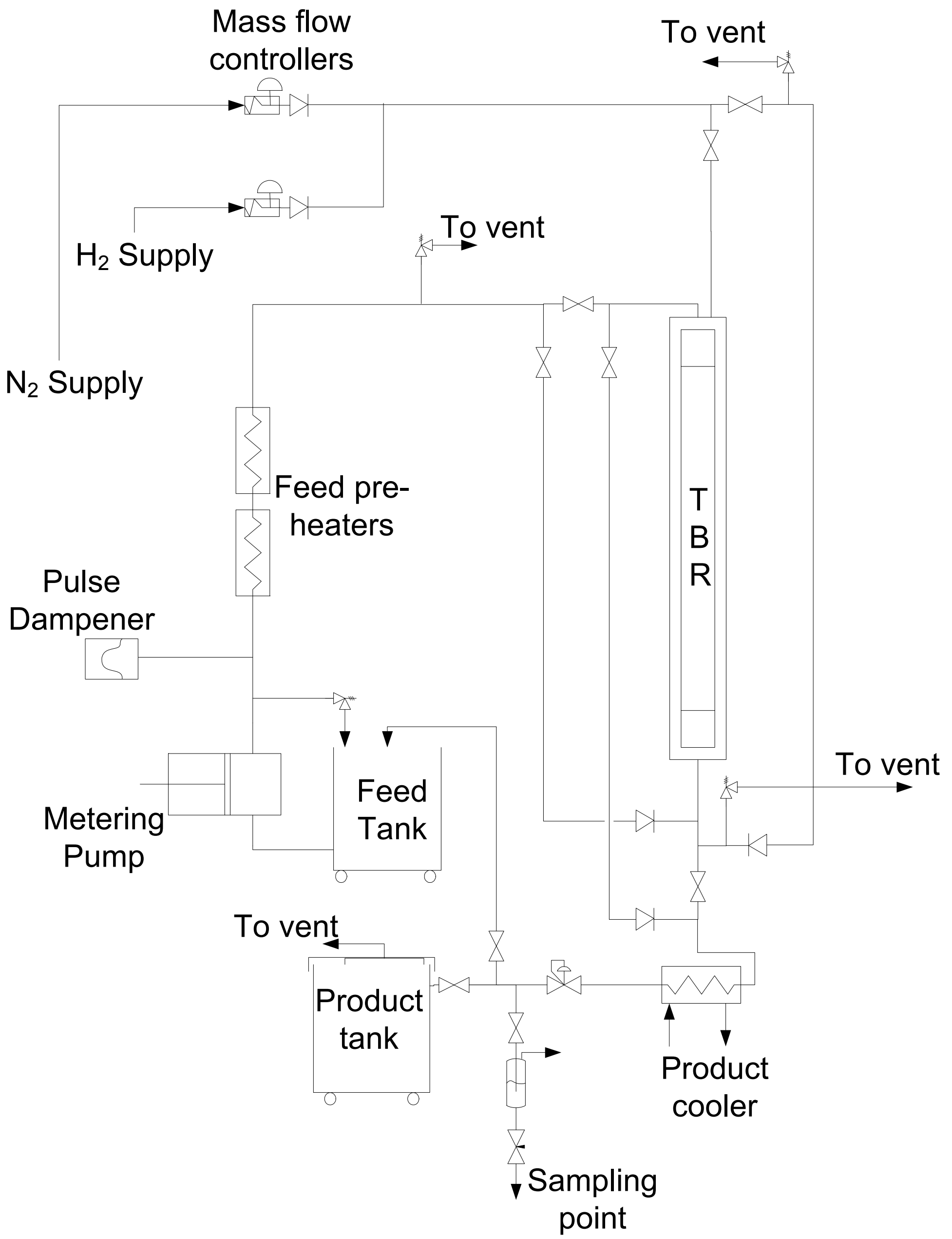

Figure 1. Schematic of the trickle-bed facility 


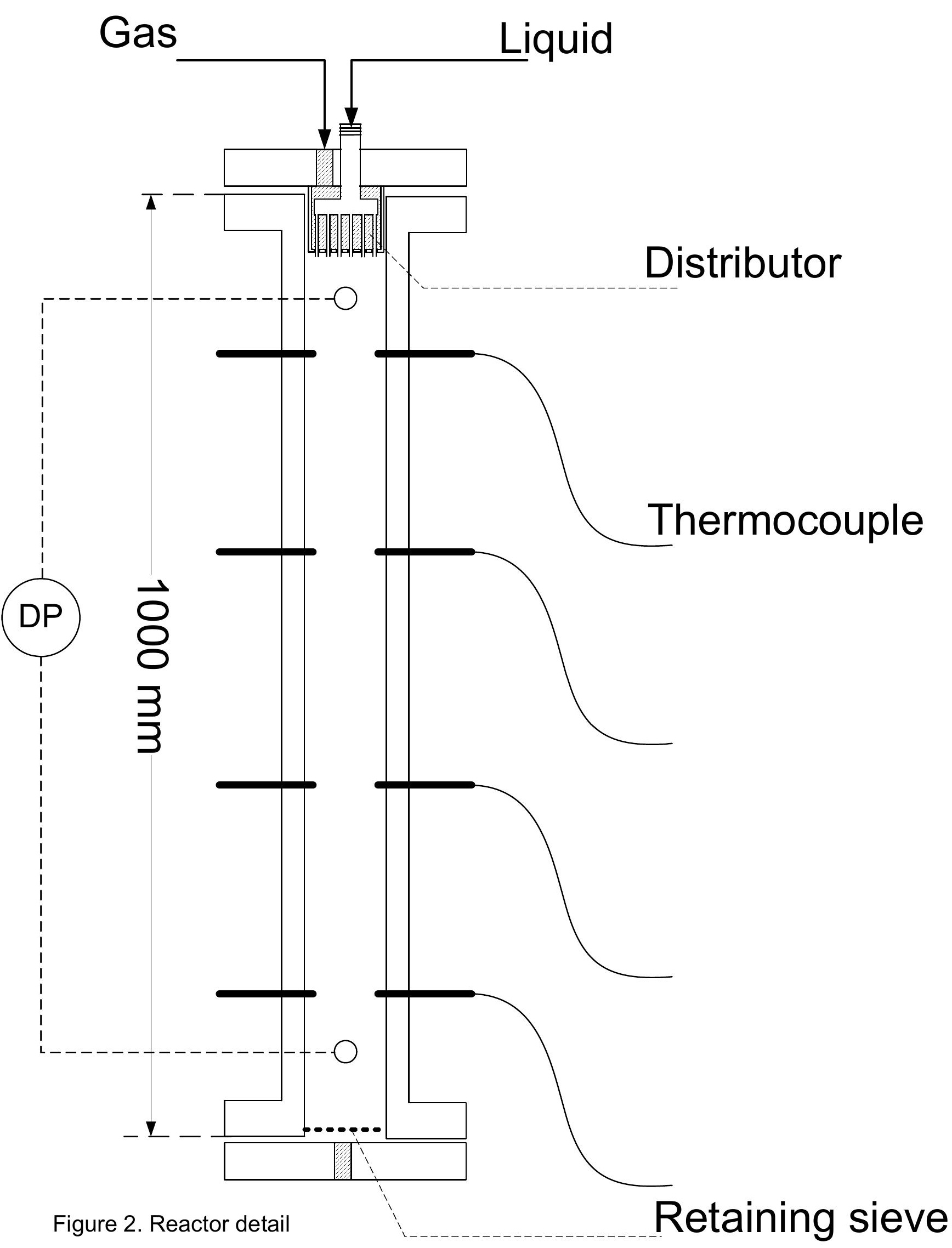




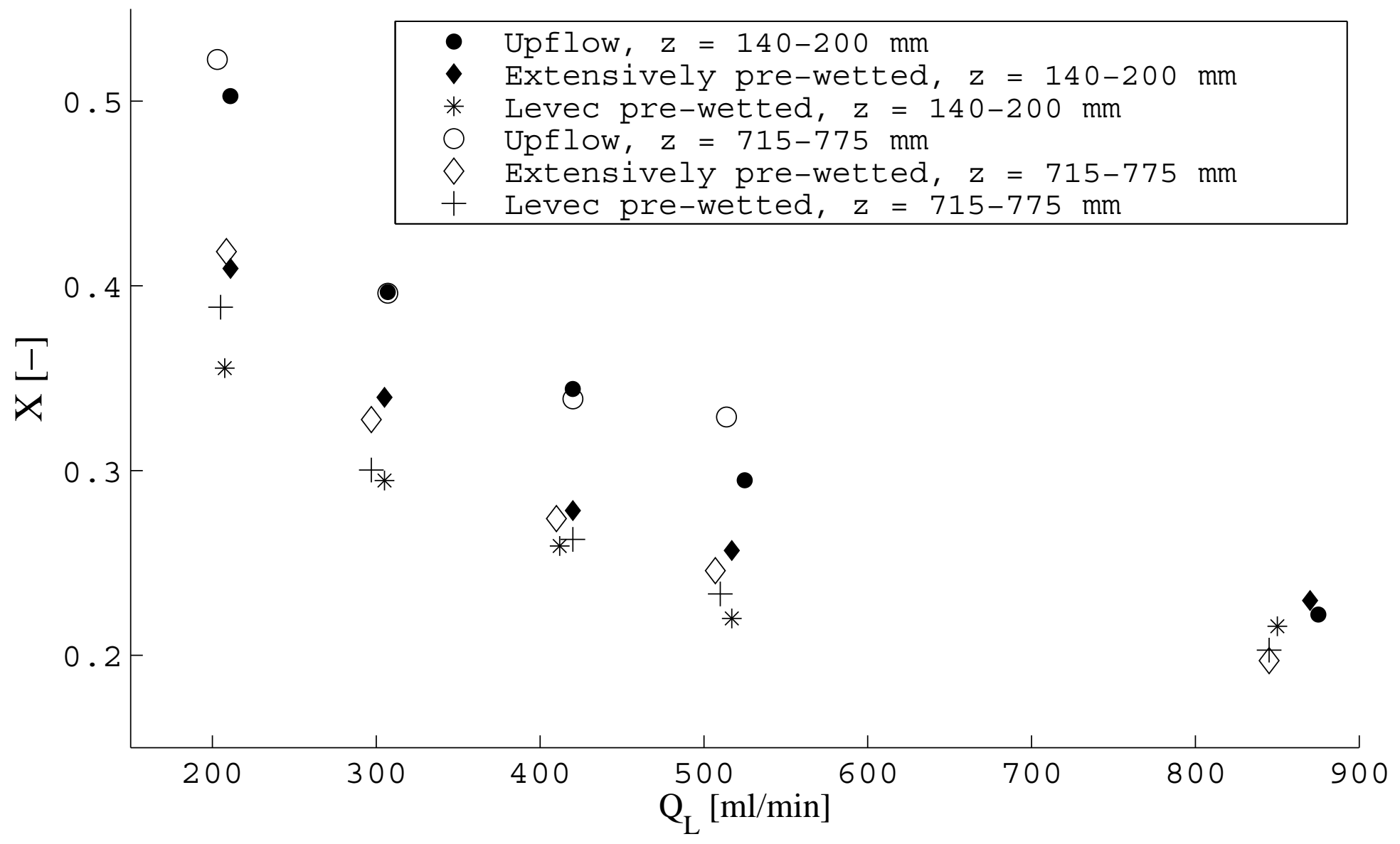




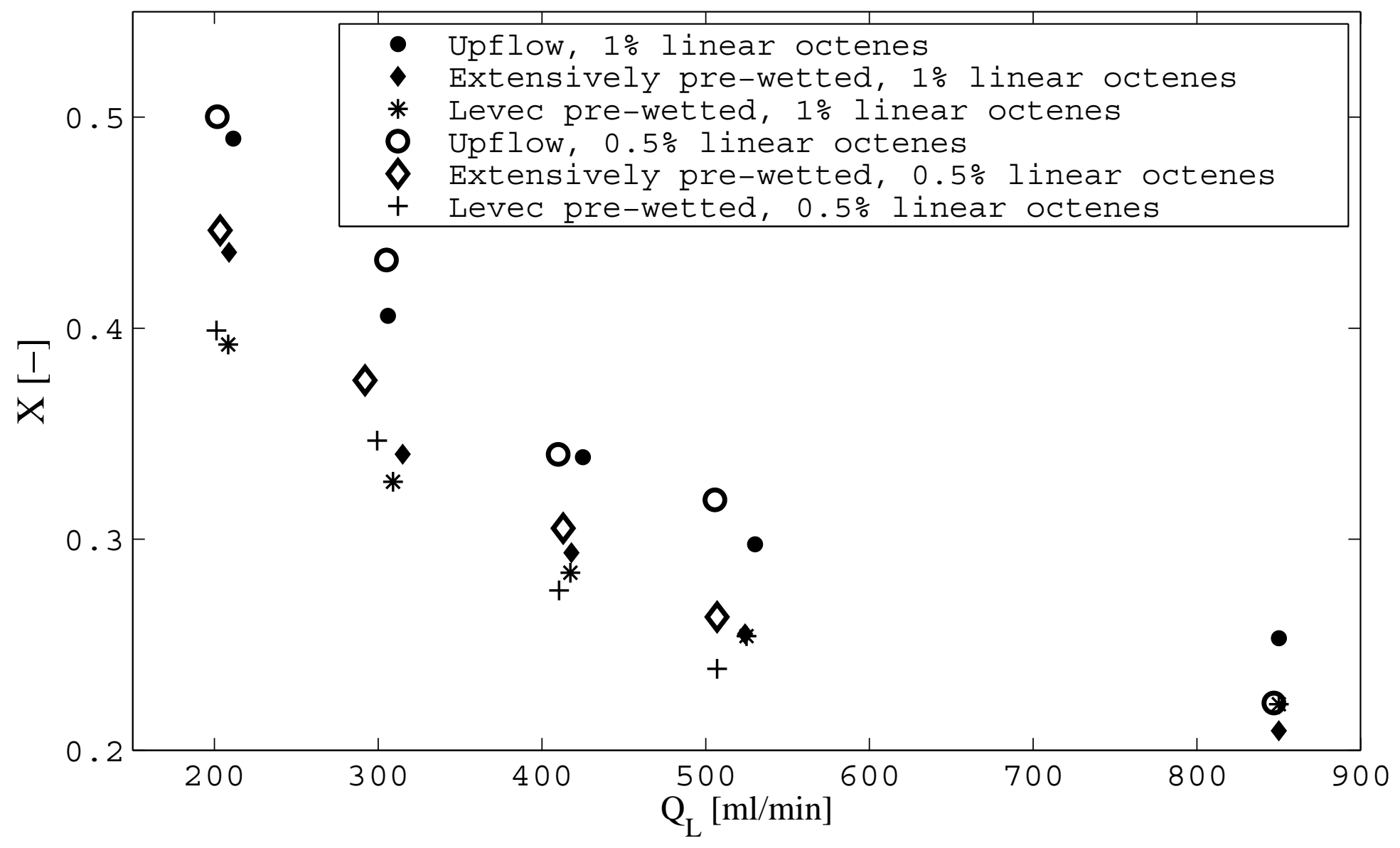


Figure 5.

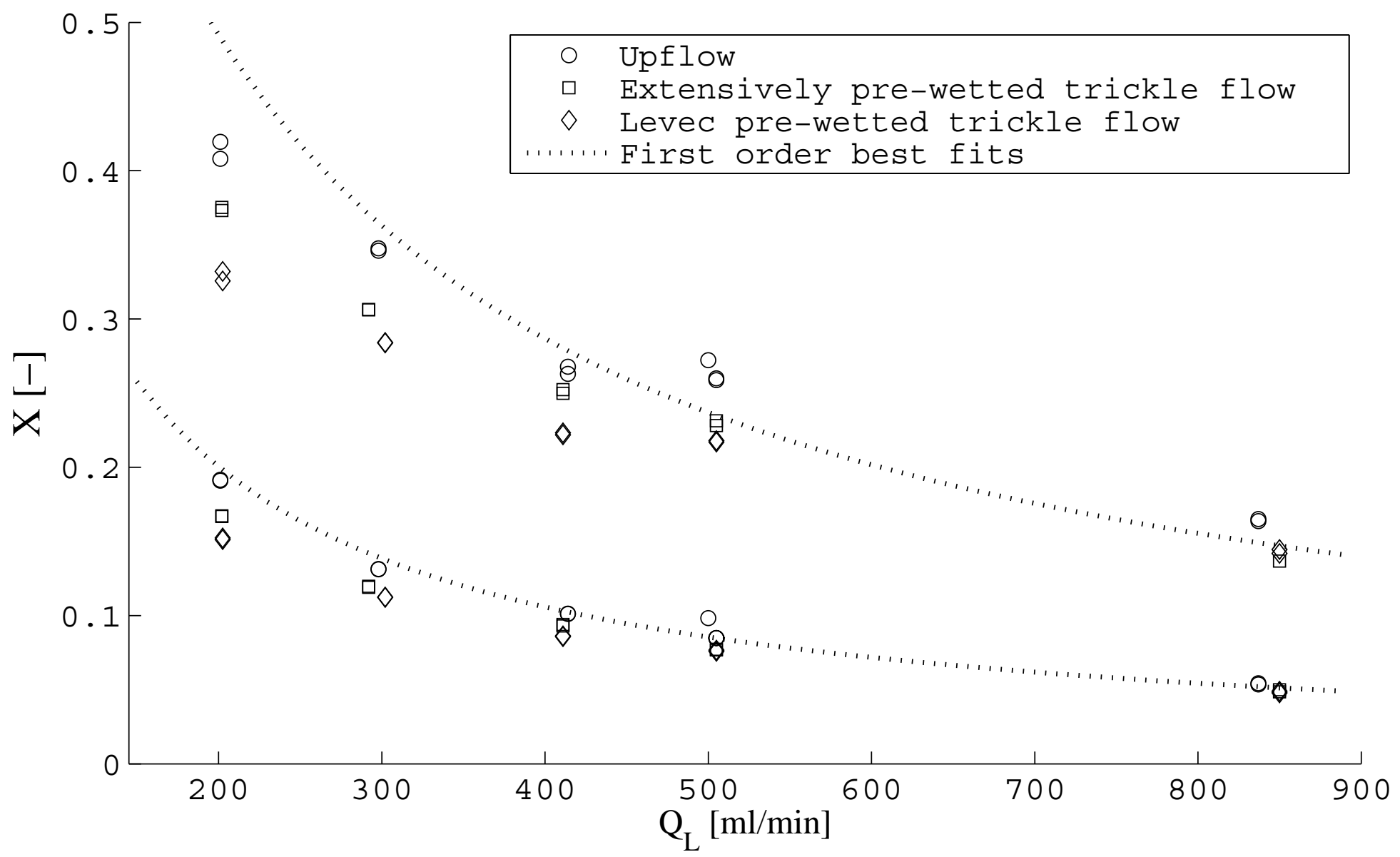


Figure 6.

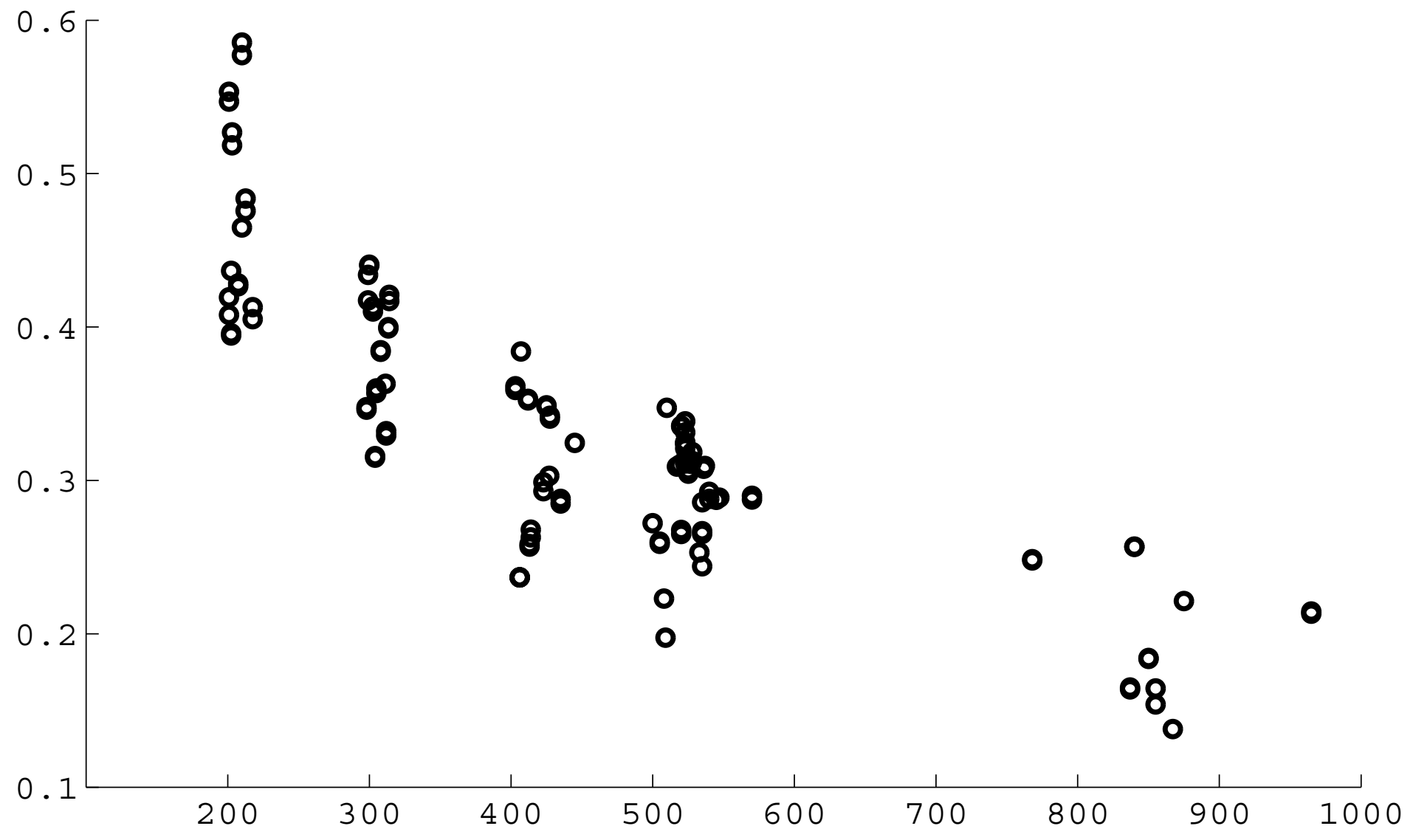


Figure 7(a).

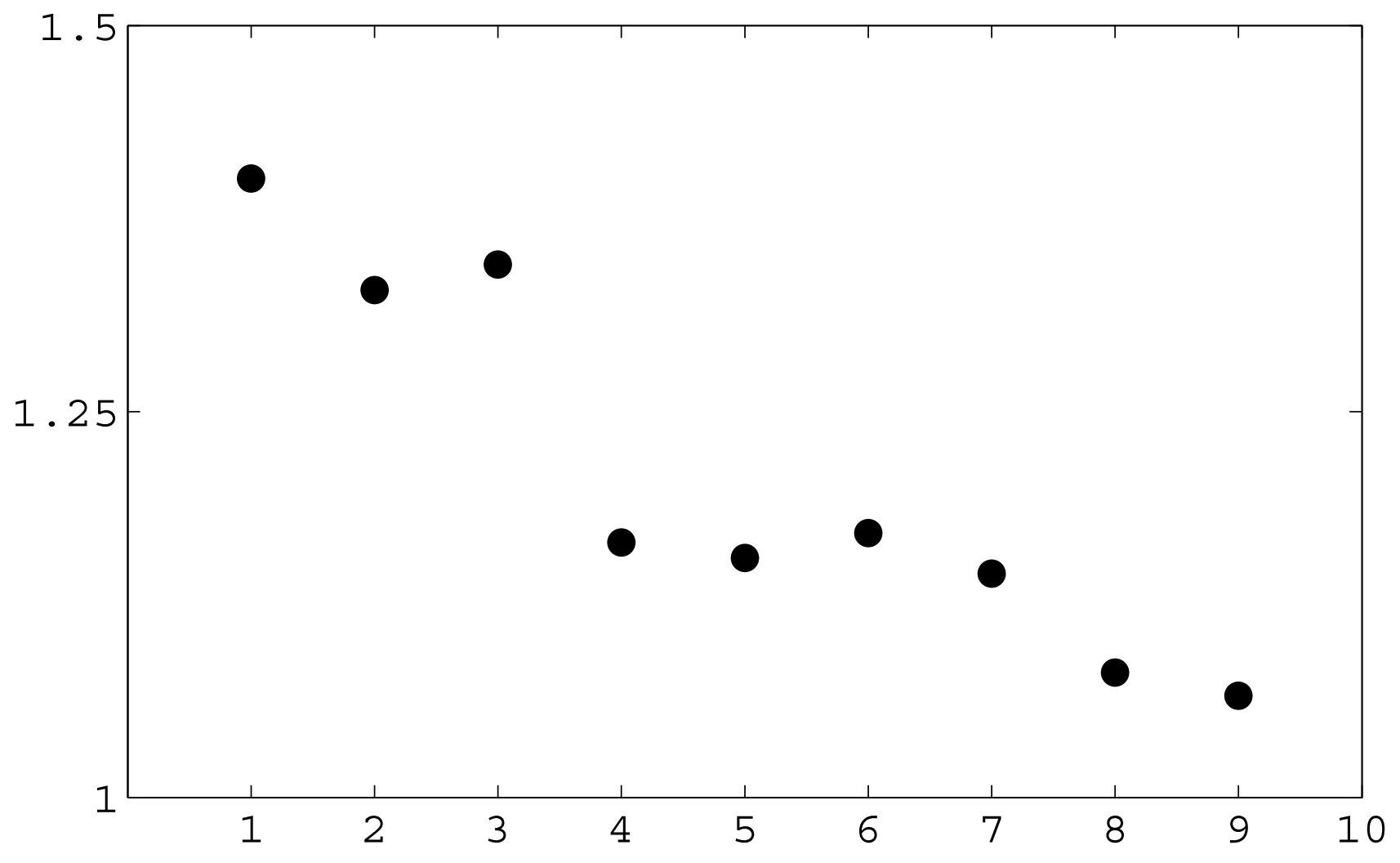


Figure $7(b)$

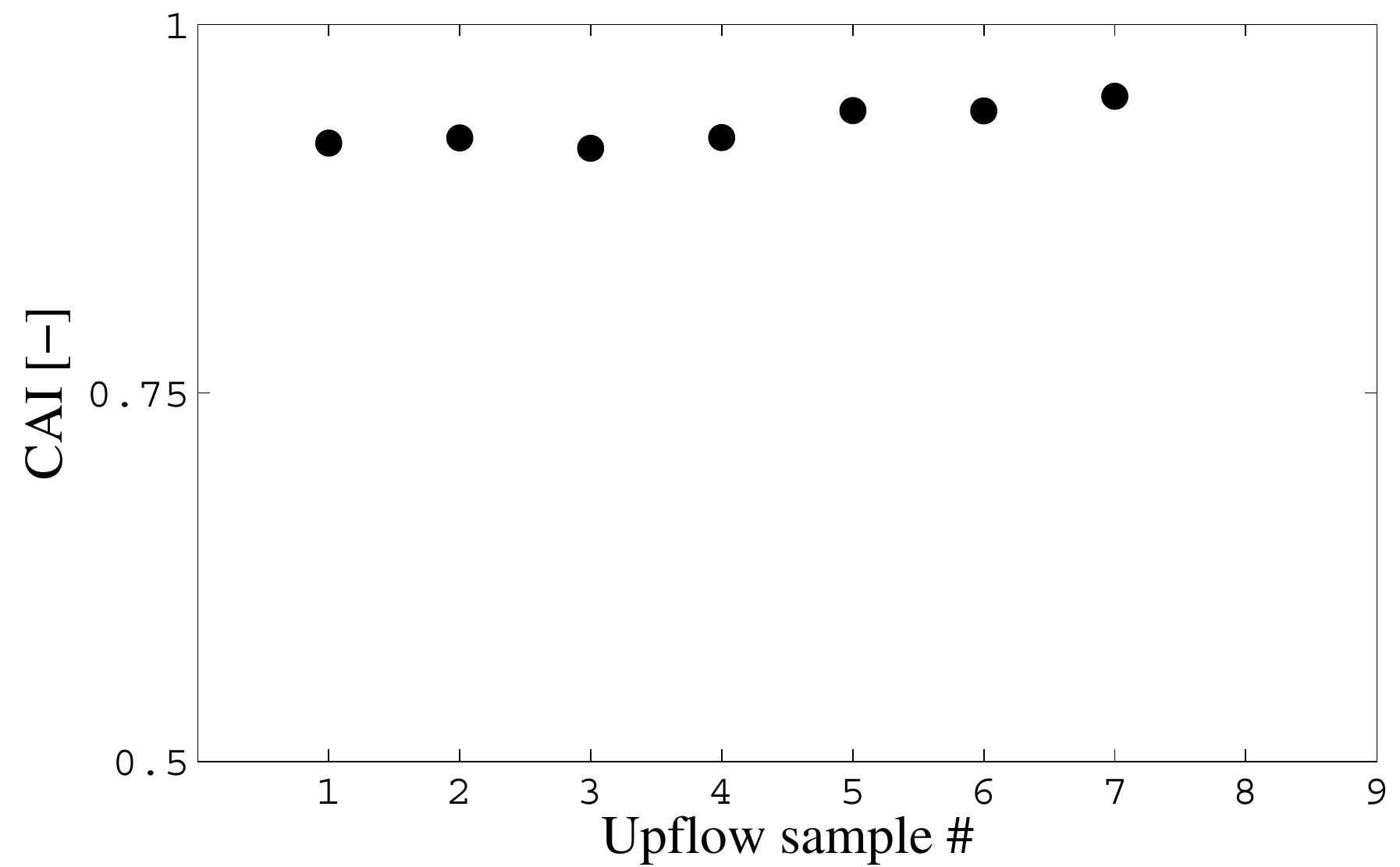


Figure 8.

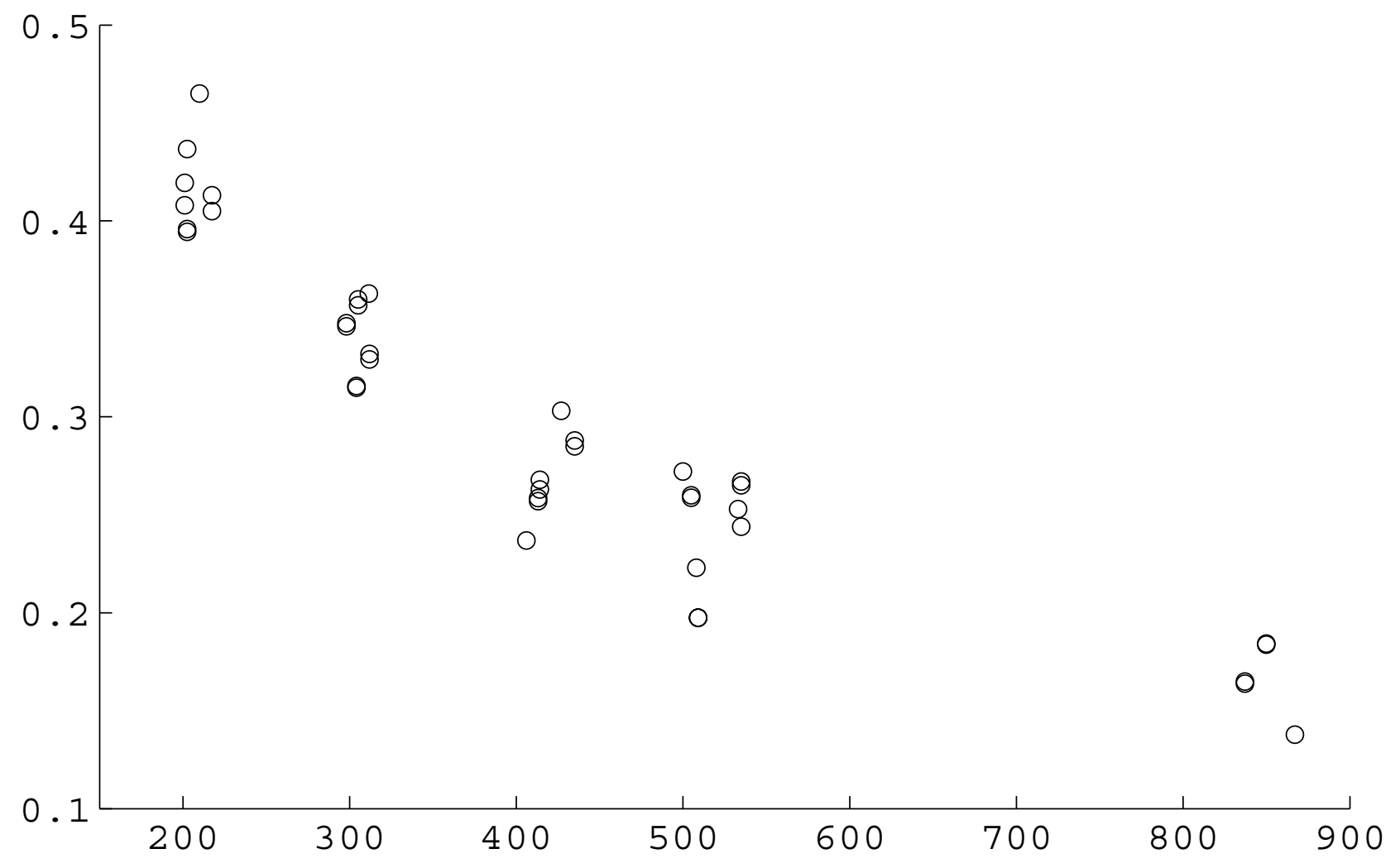


Figure 9(a).

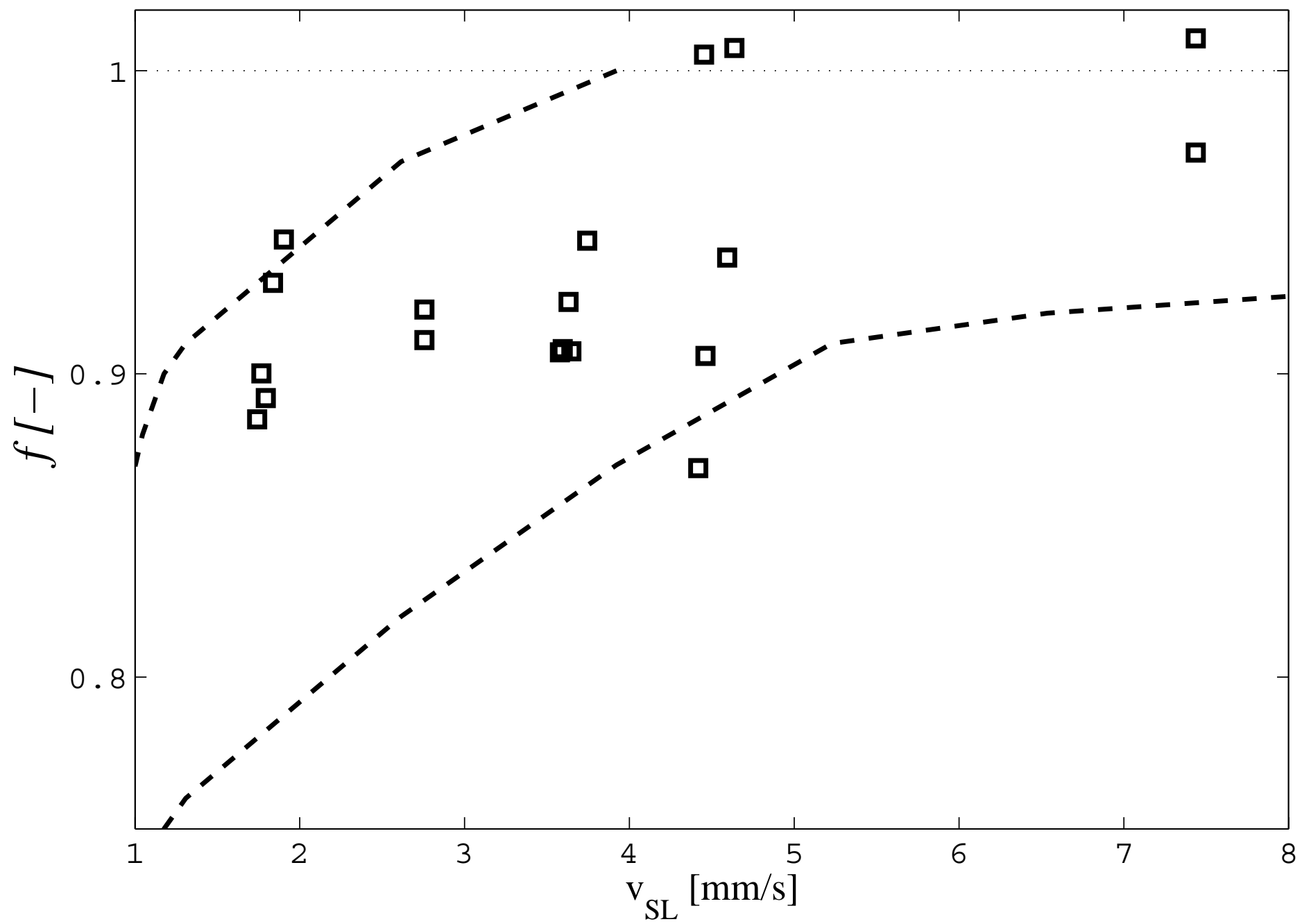


Figure 10.

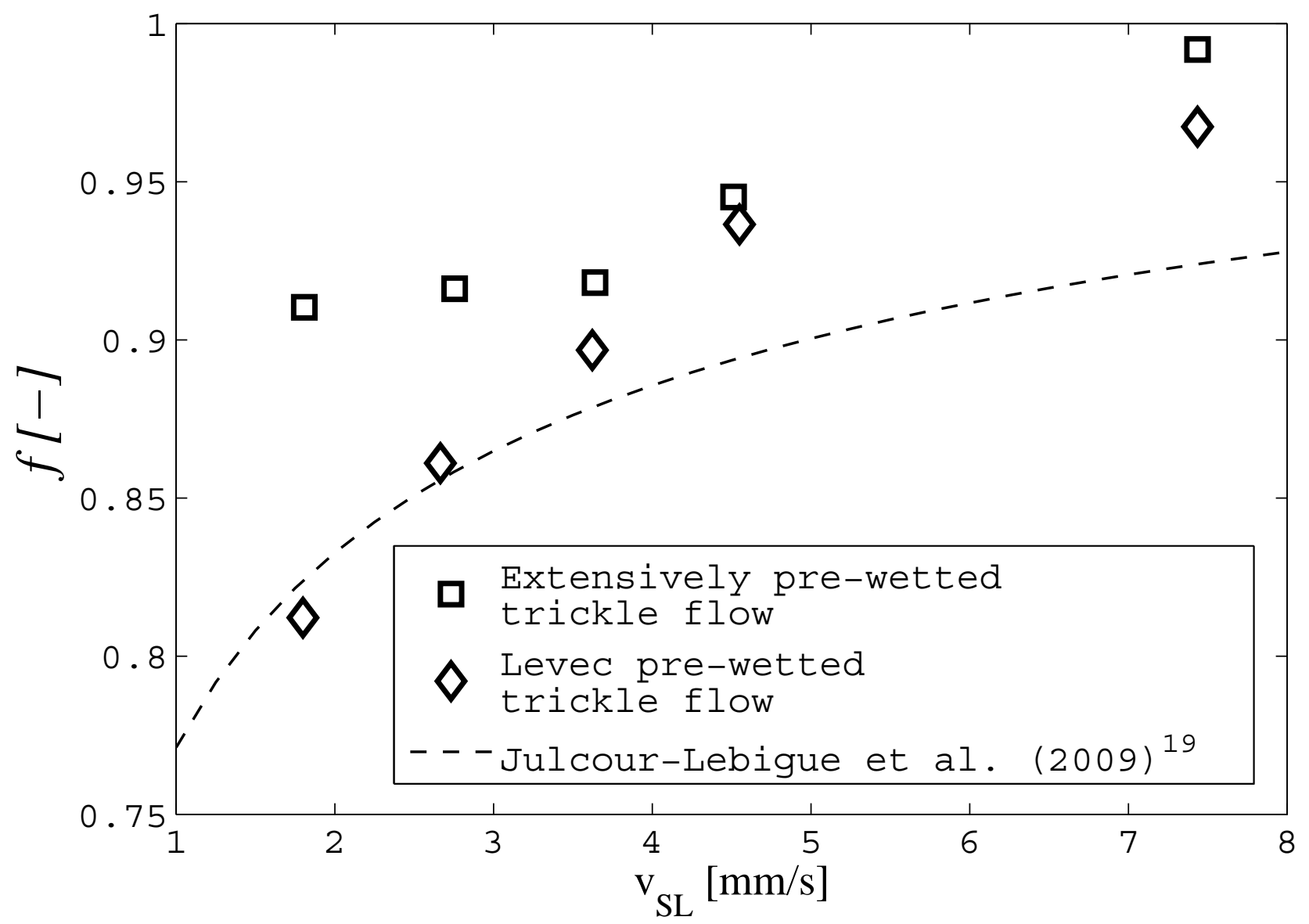


Figure 11.

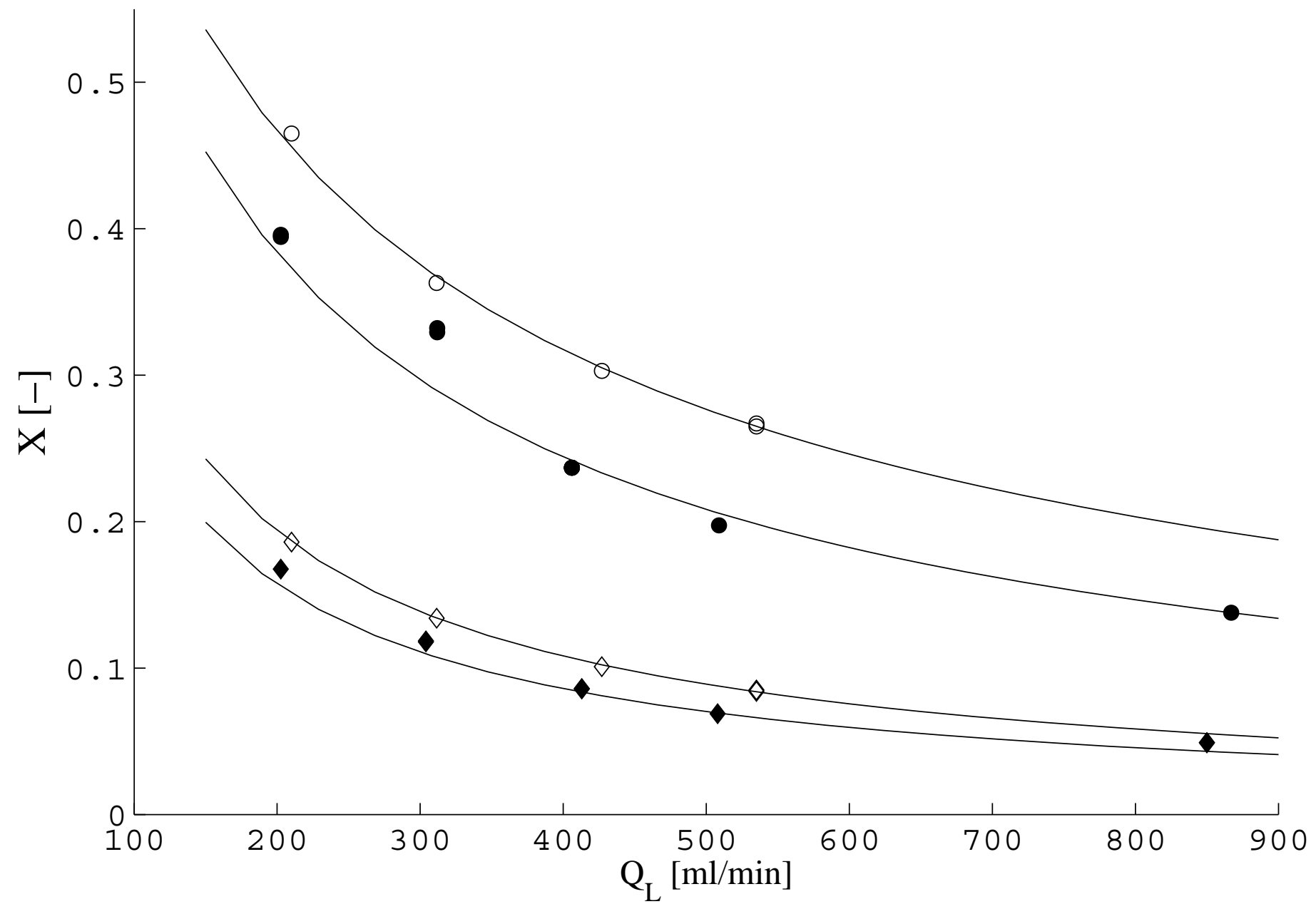


Figure 12.

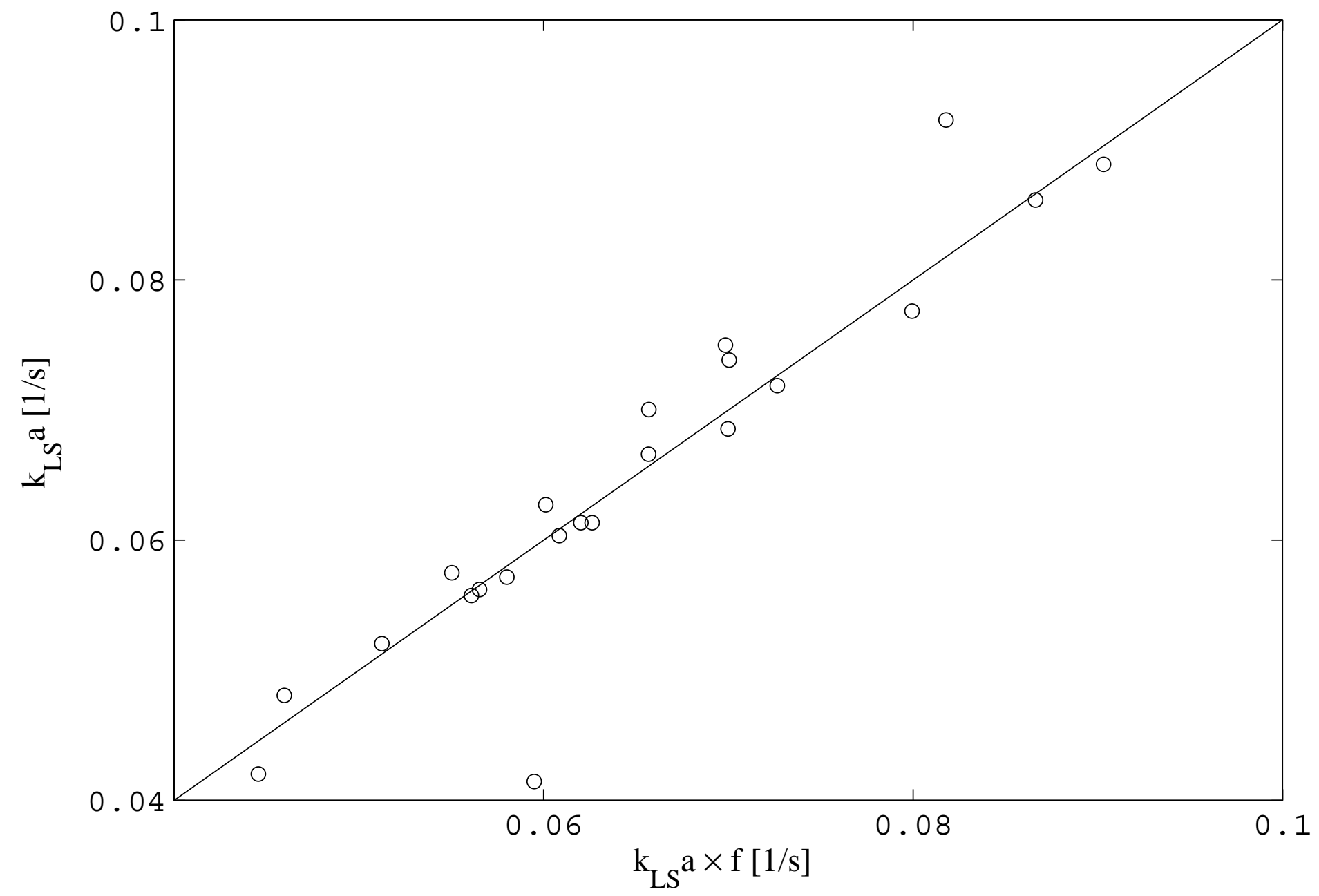


$\mathrm{v}_{\mathrm{SL}}[\mathrm{mm} / \mathrm{s}]$

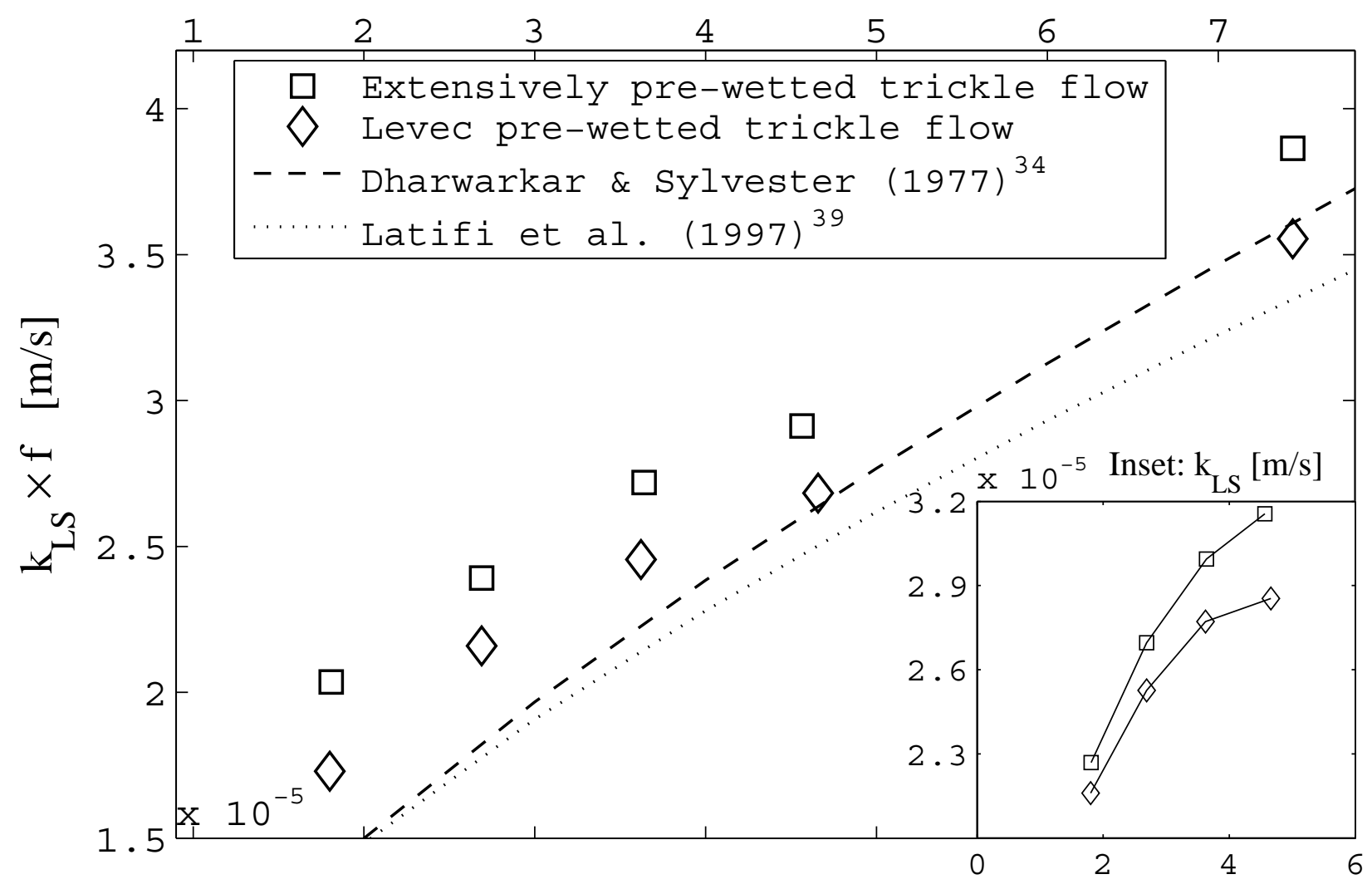


Figure 14.

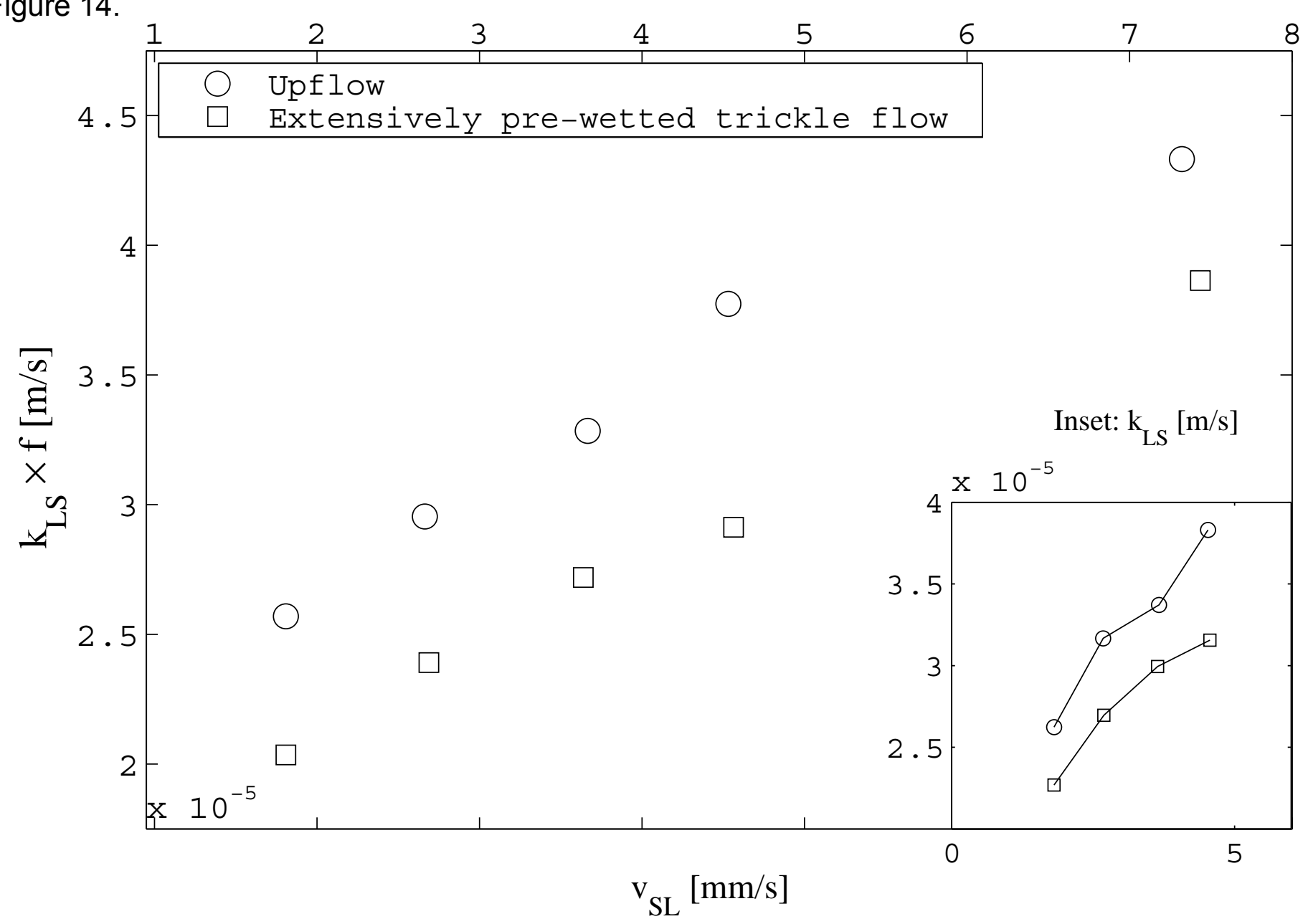


) เЈXயா वEा

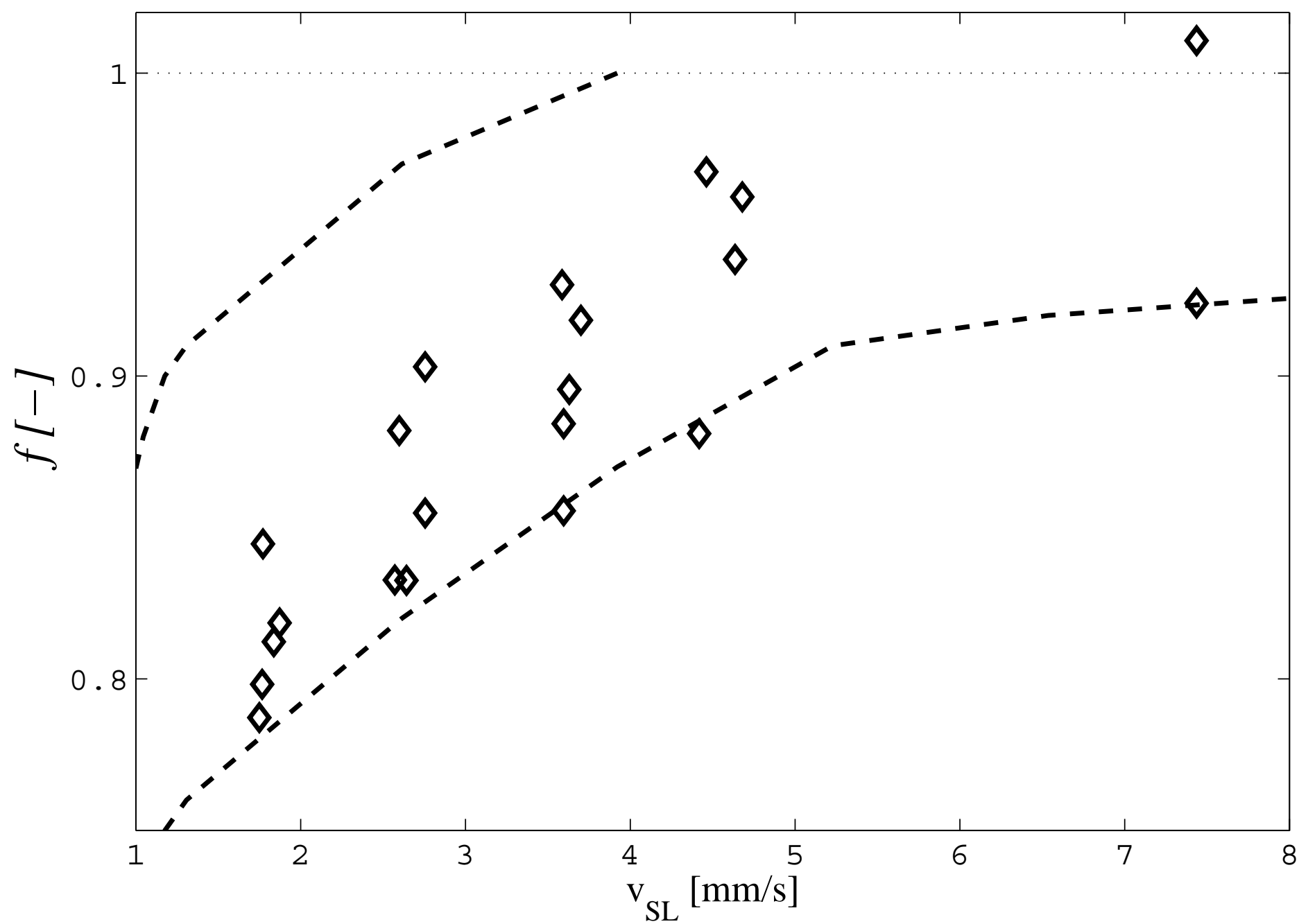

